\title{
Ganglioside GM3 Is Antiangiogenic in Malignant Brain Cancer
}

\author{
Thomas N. Seyfried and Purna Mukherjee \\ Department of Biology, Boston College, Chestnut Hill, MA 02467, USA \\ Correspondence should be addressed to Thomas N. Seyfried, thomas.seyfried@bc.edu
}

Received 17 December 2009; Revised 12 March 2010; Accepted 10 April 2010

Academic Editor: Arkadiusz Dudek

Copyright (C) 2010 T. N. Seyfried and P. Mukherjee. This is an open access article distributed under the Creative Commons Attribution License, which permits unrestricted use, distribution, and reproduction in any medium, provided the original work is properly cited.

\begin{abstract}
Progression of malignant brain tumors is dependent upon vascularity and is associated with altered ganglioside composition and distribution. Evidence is reviewed showing that the simple monosialoganglioside, GM3, possesses powerful antiangiogenic action against the highly vascularized CT-2A mouse astrocytoma, which primarily expresses complex gangliosides. Brain tumors expressing high levels of GM3 are generally less vascularized and grow slower than tumors that express low levels of GM3. GM3 inhibits angiogenesis through autocrine and paracrine effects on vascular endothelial growth factor (VEGF) and associated receptors. GM3 should be a clinically useful compound for managing brain tumor angiogenesis.
\end{abstract}

\section{Introduction}

Malignant brain cancer persists as a catastrophic illness and is the second leading cause of cancer death in children [1-4]. The failure to effectively manage malignant brain cancer has been due in large part to the highly invasive nature of the disease and to the unique anatomical and metabolic environment of the brain, which prevents the large-scale resection of tumor tissue and impedes the delivery of therapeutic drugs. Invasion/metastasis involves the dissemination of tumor cells from the primary neoplasm to surrounding tissue and distant regions. In addition, the invasive cells establish a microenvironment facilitating colonization (angiogenesis and further proliferation), resulting in macroscopic malignant secondary tumors $[5,6]$. Tumor cell invasion is correlated with tumor angiogenesis (vascularity), as prognosis is generally worse for brain tumors that are more vascular than for those that are less vascular [7-9]. Consequently, therapies that can simultaneously target both angiogenesis and invasion could provide effective longerterm management of malignant brain cancer.

\section{Glycosphingolipids and Angiogenesis}

Gangliosides are a family of cell surface-enriched glycosphingolipids that have long been implicated in tumorigenesis [10-12]. These molecules contain an oligosaccharide head group attached to a lipophilic ceramide, consisting of a sphingosine base and a long-chain fatty acid (Figure 1). The presence of sialic acid (N-acetylneuraminic acid, NeuAc) distinguishes the gangliosides from other glycosphingolipids. Gangliosides are anchored in the outer surface of plasma membranes through their ceramide moiety, which allows the head group to modulate numerous cell surface events such as growth, migration, adhesion, and signaling [12-15].

The structurally simple monosialoganglioside GM3 contains a single terminal sialic acid (Figure 1). Nacetylneuraminic acid is the predominant sialic acid species expressed in mammalian brain gangliosides $[16,17]$. In contrast to $\mathrm{N}$-acetylneuraminic acid, $\mathrm{N}$-glycolylneuraminic acid is a predominant sialic acid species expressed in gangliosides from nonneural tissues of most nonhuman species (rodents, bovine, etc.) [17]. As humans lack the gene for the synthesis of $\mathrm{N}$-glycolylneuraminic acid $[18,19]$, expression of $\mathrm{N}$-glycolylneuraminic acid in gangliosides of human cells or tissues is attributed to contamination from exposure to nonhuman serum or from diet [17, 20, 21]. The involvement of gangliosides in angiogenesis is dependent on the intact molecules as neither asialo species nor sialic alone influence angiogenesis [22].

GM3 modulates the function of several receptors implicated with angiogenesis to include those for the insulinlike growth factor-1 (IGF-1), basic fibroblast growth factor (b-FGF), epidermal growth factor (EGF), platelet-derived 


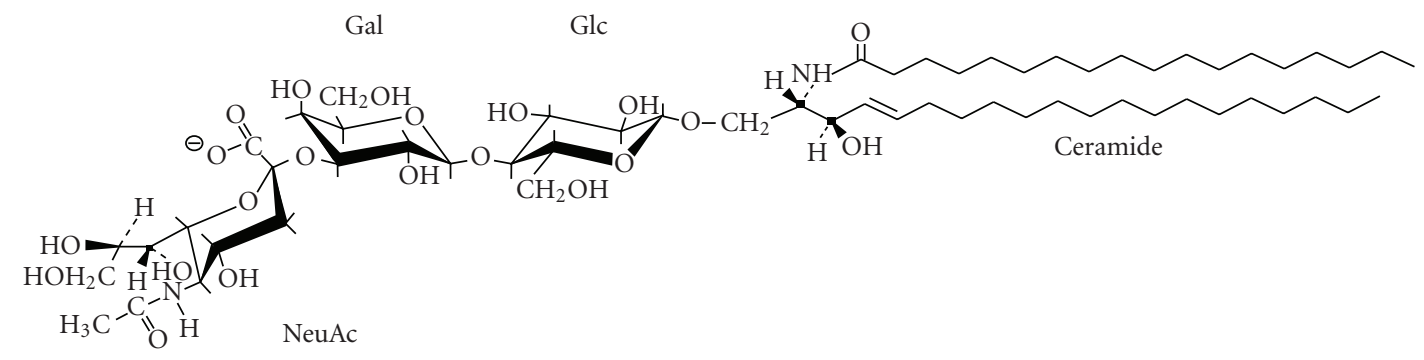

Figure 1: Structure of Ganglioside GM3 (NeuAc-alpha $2 \rightarrow$ 3Gal-beta1 $\rightarrow$ 4Glc-beta1 $\rightarrow$ 1' Ceramide) (from [14] with permission).

TABLE 1: Association of GM3 levels to the vascularity of experimental brain tumors*.

\begin{tabular}{llcc}
\hline Brain Tumors & GM3 & Canglioside Distribution & Vascularity \\
Complex Gangliosides & \\
EPeuse & High & Low & Low \\
CBT-1 & High & Low & Low \\
CBT-3 & High & Low & Low \\
\hline CBT-4 & Low & high & High \\
CT-2 & Low & High & High \\
CT-2A & Low & High & High \\
\hline Human & & & \\
U87MG & Low & High & High \\
\hline
\end{tabular}

*All mouse brain tumors were produced from implantation of 20methylcholantherene into the ventricle (EPEN), the cerebrum (CT), or the cerebellum (CBT) of C57BL/6J mice as we previously described [43].

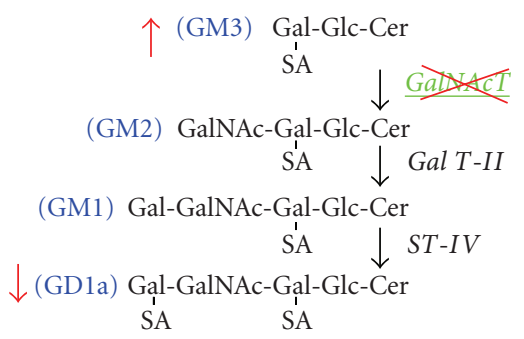

Figure 2: Pathway for the synthesis of ganglioside GM2 from GM3 by GalNAc-T. GalNAc-T adds a beta-linked $N$-acetylgalactosamine residue to the galactose of GM3 to form GM2, a key step required for the synthesis of complex gangliosides, GM2, GM1, and GD1a. Antisense targeting of the GalNAc-T gene reduces GDla content, while increasing GM3 content [7].

growth factor (PDGF), vascular endothelial growth factor (VEGF), and cell adhesion molecules including the integrins $[7,12,23-28]$. GM3 also reduces proliferation and enhances apoptosis of rapidly proliferating neural stem cells [29]. Furthermore, Alessandri, Ziche, and coworkers originally found that several complex gangliosides (GM2, GM1, GD3, GD1a, GD1b, and GT1b) enhanced the action of angiogenic inducers, whereas ganglioside GM3 was inhibitory [30-32]. These observations suggest that GM3 could have therapeutic potential against tumor cell proliferation and angiogenesis.

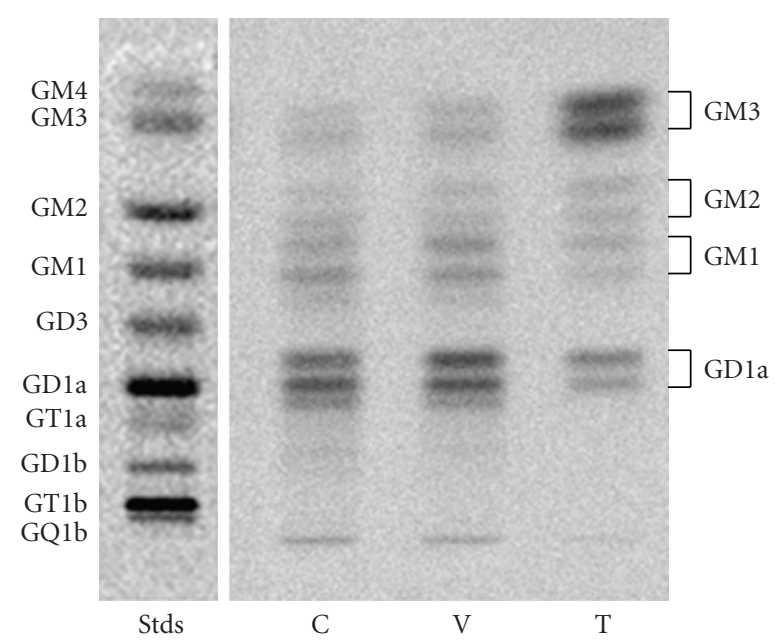

FIGURE 3: High-performance thin-layer chromatographic analysis of ganglioside distribution in the CT-2A astrocytoma: Control untransfected CT-2A (C), CT-2A transfected with empty vector alone $(\mathrm{V})$, and CT-2A transfected with the antisense sequence to the GalNAc-T gene (T). Synthesized gangliosides appear as double bands due to ceramide structural heterogeneity. Analysis of synthesized gangliosides and standards (left lane) was as we described [7]. Knockdown of the GalNAc-T gene elevated GM3 content, while reducing GDla content in the antisense T cells.

The ratio of GM3 to the proangiogenic gangliosides GD3 and GD1a (GM3/GD3; GM3/GD1a) is lower in more metastatic and aggressive tumors than that in less metastatic tumors [7, 33-35], suggesting that elevated expression of complex gangliosides enhances tumor malignancy. In contrast to most human glioma tumor tissues, which contain high levels of the pro-angiogenic ganglioside GD3 [36-41], GD3 is not heavily expressed in mouse brain tumors or in most cultured human brain tumor cells [17, 42-44]. Although GM3 is also expressed in malignant human brain tumors, we think that GM3 expression in these tumors might serve to regulate or to counteract the pro-angiogenic action of GD3 and other complex gangliosides.

\section{Evidence Supporting the Anti-Angiogenic Action of GM3 in Brain Cancer}

Table 1 summarizes data from our previous studies on the association of GM3 expression with the angiogenic 


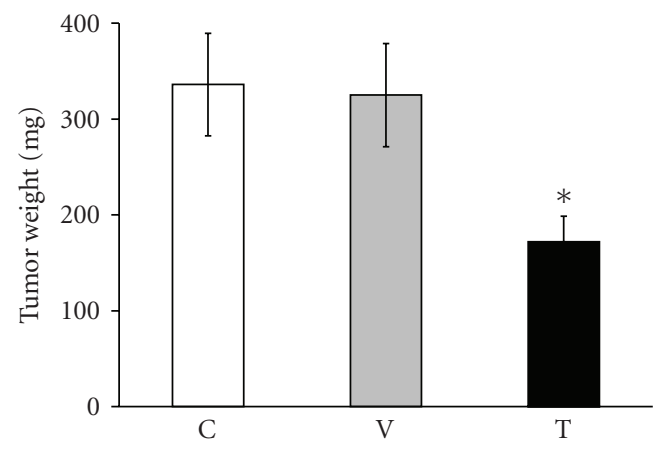

(a)
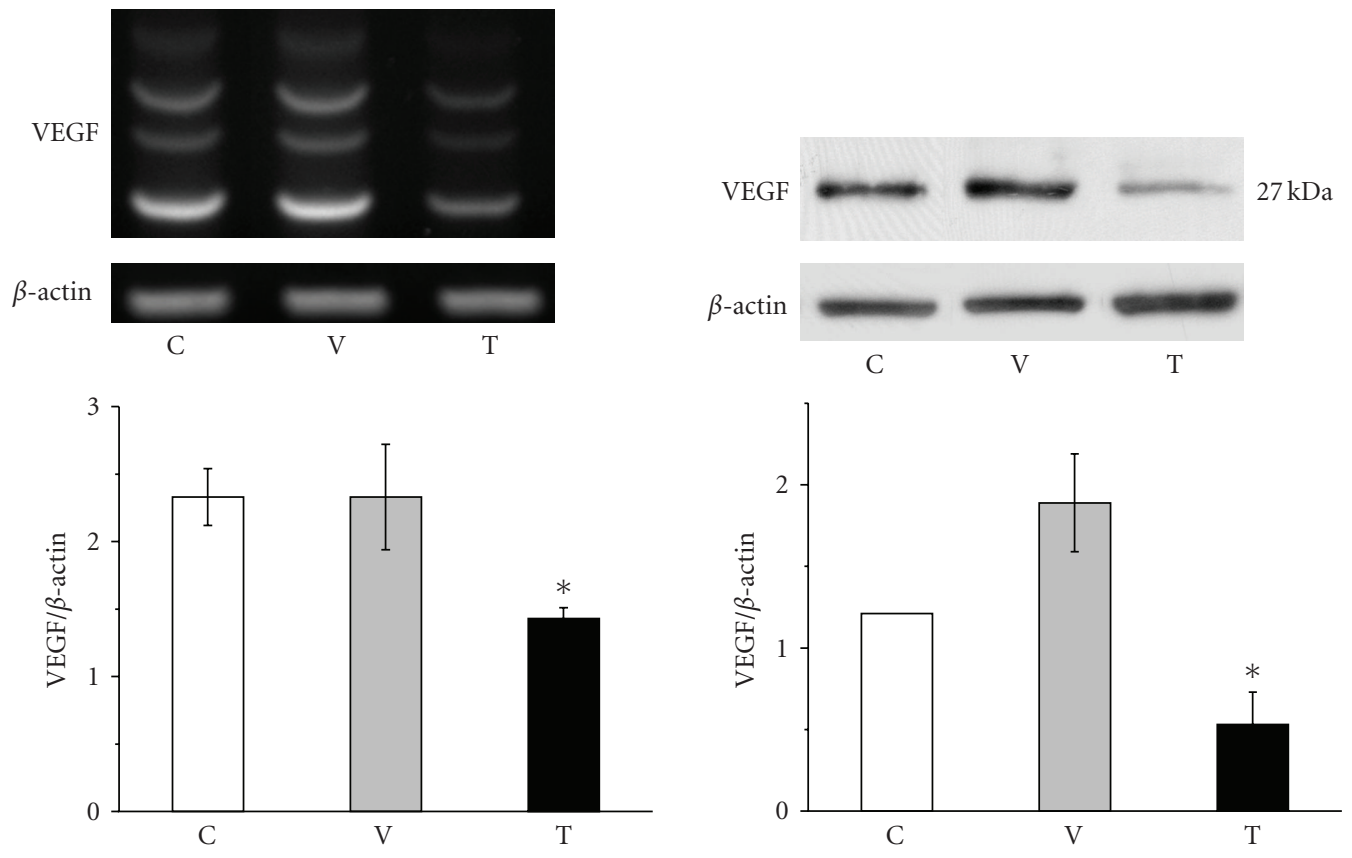

(b)

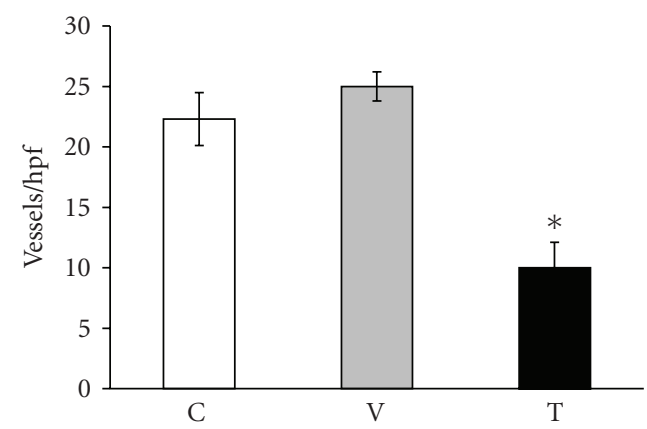

(c)

FIGURE 4: Ganglioside shift reduces growth, VEGF gene and protein expression, and vascularity in the CT-2A astrocytoma: Control untransfected CT-2A (C), CT-2A transfected with empty vector alone (V), and CT-2A transfected with the antisense sequence to the GalNAc$T$ gene (T). (a) The values are expressed as mean mg wet weight \pm SE. ${ }^{*}$ : significant compared to control $\mathrm{C}$ and $\mathrm{V}$ tumors at the $P<.01$ level. CT-2A $(n=10)$, CT-2A/V $(n=12)$, and CT-2A/TNG $(n=14)$ independent tumors. (b) RT-PCR and Western blot for VEGF. Other details are in [7]. (c) Vascularity determined using factor VIII-immunostained microvessels per $\times 200$ field (hpf, high-powered field) from tissue sections as we described [7]. 


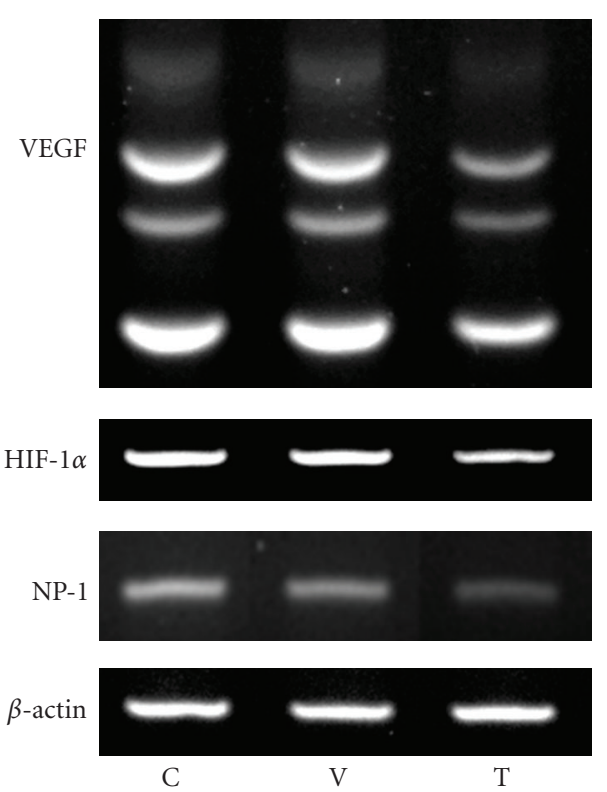

(a)

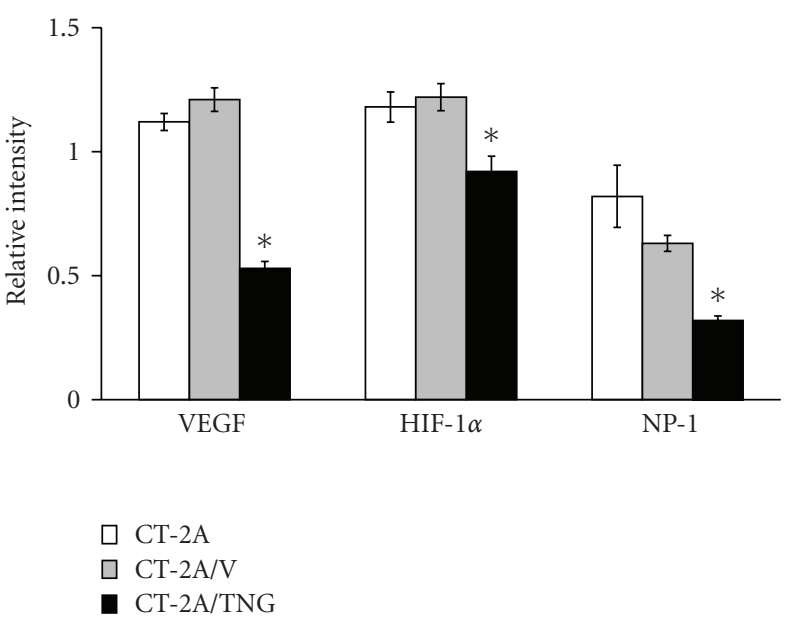

(b)

FIGURE 5: Ganglioside shift reduces VEGF, HIF-1 $\alpha$, and NP-1 gene expression in CT-2A- cultured cells: Control untransfected CT-2A (C), CT-2A transfected with empty vector alone (V), and CT-2A transfected with the anti-sense sequence to (a) the GalNAc-T gene (T). VEGF (multiple splice variants: 400-600 bp), HIF-1 $\alpha$ (365 bp), and NP-1 (551 bp) amplification products were detected in each tumor cell line. Experimental conditions are as we described [7]. The gene to $\beta$-actin levels are expressed as the means of three independent samples \pm SE. ${ }^{*}$ : Significant compared to control $\mathrm{C}$ and $\mathrm{V}$ cells at (b) the $P<.01$.

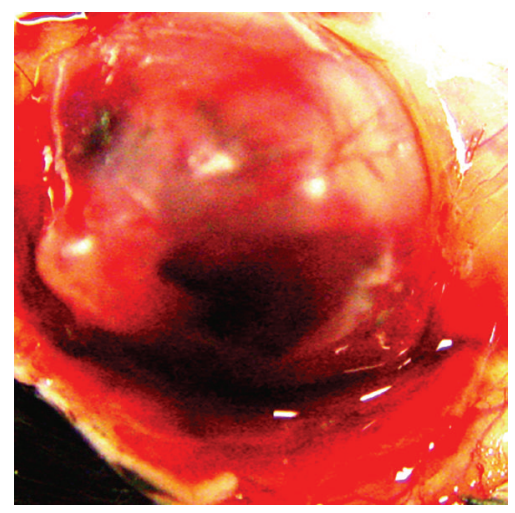

Control

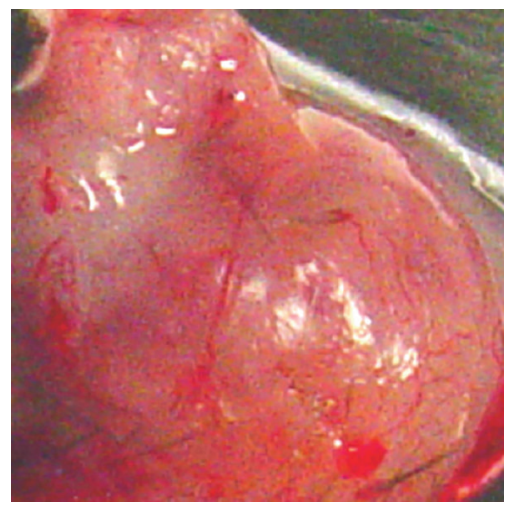

GM3 treated

FIGURE 6: GM3 reduces CT-2A tumor vascularity when added to the tumor microenvironment. Small fragments of the CT-2A tumor were grown in Matrigel that contained either no GM3 (control) or GM3 $(40 \mu \mathrm{M})$. The tumor was grown in Matrigel for approximately two weeks in the flank of the syngeneic host C57BL/6 mice according to our standard procedures $[7,34]$. Florid vascularization and the number and size thrombotic vessels were noticeably less in the presence than in the absence of GM3. Similar results were found in two independent experiments.

properties of multiple experimental mouse and human brain tumor models [17, 44, 45]. This survey shows that brain tumors with high GM3 expression are less angiogenic (vascularized) than brain tumors with low GM3 expression. GM3 expression was also correlated with greater cell-cell adhesion and slower growth [14, 44]. We later showed that the gene-linked knockdown of GM3 expression in the experimental ependymoblastoma (EPEN) tumor, which contains GM3 as the only ganglioside, increased vascularity (angiogenesis) [34]. An opposite effect was observed in the highly angiogenic CT-2A astrocytoma when we upregulated GM3 expression (below). These and other findings led us to conclude that the ratio of GM3 to complex gangliosides (GM1, GD1a, GT1b) can influence the angiogenic properties 


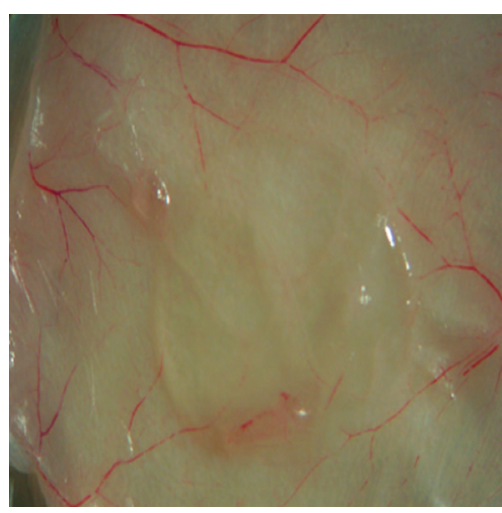

Control

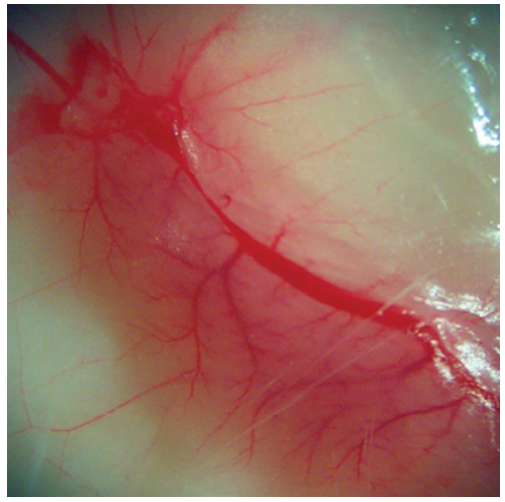

GDla $(40 \mu \mathrm{M})$

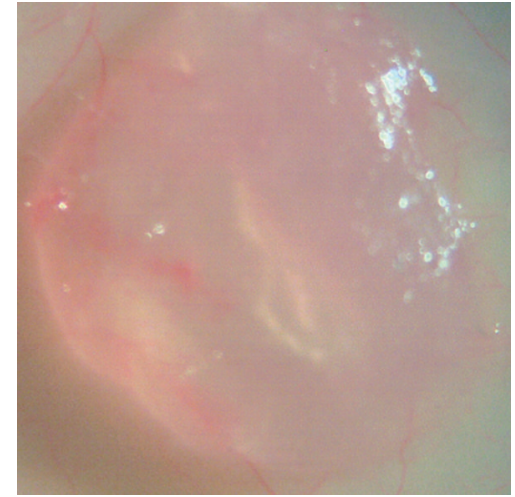

GM3+GD1a $(40 \mu \mathrm{M}$ each $)$

FIGURE 7: GM3 inhibits the pro-angiogenic effects of GD1a in the in vivo Matrigel assay. Matrigel alone (control) or containing GD1a or GD1a with GM3 was injected subcutaneously (s.c.) in SCID mice as we described [49]. Plugs were photographed (12.5×) on day 7 after Matrigel injection.

of a broad range of brain tumor types and are consistent with previous findings on the role of GM3 in other systems $[22,29,32,41,46-49]$.

\section{Anti-Angiogenic Action of GM3 in the CT-2A Astrocytoma}

The CT-2A astrocytoma was produced following implantation of the chemical carcinogen, 20-methylcholanthrene, into the cerebral cortex of C57BL/6J mouse according to the procedures of Zimmerman and Arnold [44, 50]. The CT-2A tumor grows rapidly, is deficient in the phosphatase and tensin homologue/tuberous sclerosis complex 2 , and is highly angiogenic $[7,51,52]$. We used an antisense construct to inhibit GalNAc-T expression in CT-2A cells as shown in (Figure 2). This caused a significant shift in ganglioside distribution, elevating GM3 content while reducing GD1a content (Figure 3).

The shift in ganglioside distribution significantly reduced growth, VEGF gene and protein expression, and blood vessel density in the orthotopically grown CT-2A tumors (Figure 4). Moreover, the shift in ganglioside distribution reduced gene expression for hypoxia inducible factor 1a (HIF- $1 \alpha$ ) and the VEGF coreceptor neruropilin-1 (NP-1) in the CT-2A cultured cells (Figure 5). This is interesting as HIF- $1 \alpha$ is a transcription factor that regulates VEGF expression through the PI-3k/Akt signaling pathway [51, 5355]. Viewed collectively, these data show that endogenous upregulation of GM3 reduces growth and angiogenesis in the rapidly growing and highly vascularized CT-2A mouse astrocytoma.

It was initially unclear, however, whether it was the elevation of GM3, the reduction of the pro-angiogenic ganglioside GD1a, or the change in GM3/GD1a ratio that was responsible for the reduction in CT-2A angiogenesis. It is well documented that gangliosides are shed from tumor cells into the microenvironment where stromal (endothelial) cells take them up to influence tumor progression [56-
60]. Our most recent findings show that GM3, by itself, markedly reduces CT-2A vascularity when grown in the in vivo Matrigel model (Figure 6). These findings suggest that GM3 could be applied as a drug therapy directly to the tumor site and to surrounding areas following surgical tumor resection in humans. Alternatively, GM3 could be applied in liposomes as a pharmacotherapy for preformed tumors. Our findings in brain tumor cells are also consistent with previous findings in rabbit cornea showing that GM3 applied directly to tissue is antiangiogenic [32]. Viewed, collectively, our findings indicate that GM3 has powerful anti-angiogenic action against the CT-2A astrocytoma when present in the microenvironment and can counteract the pro-angiogenic effects of complex gangliosides.

Further evidence for a direct anti-angiogenic role of GM3 came from our recent studies with human umbilical vein endothelial cells, HUVEC. We found that GM3, by itself, significantly suppresses VEGF-induced proliferation and migration of HUVEC [49]. Moreover, GM3 significantly blocks GD1a-induced angiogenesis in the in vivo Matrigel assay (Figure 7). GDla is a complex ganglioside associated with enhanced angiogenesis $[7,61]$. The suppression of VEGF receptor 2 and Akt phosphorylation underlies the anti-angiogenic effect of GM3 on HUVEC (Figure 8). Additionally, the EPEN tumor, which expresses only GM3, has few blood vessels relative to tumors that express complex gangliosides [44, 45]. Consistent with our findings, Chung and coworkers recently showed that GM3 could suppress angiogenesis through the inactivation of VEGF-induced signaling by direct interaction with VEGFR-2 [47]. GM3 treatment could also reduce in vivo vascularity in the Lewis lung carcinoma model [47], while van Cruijsen et al. showed that vascularity was less and patient survival was better for nonsmall cell lung carcinomas that contained more GM3 than less GM3 [62]. Hence, GM3 is anti-angiogenic through its inhibition of the proangiogenic actions of complex gangliosides as well as through its direct inhibition of endothelial cell growth. 


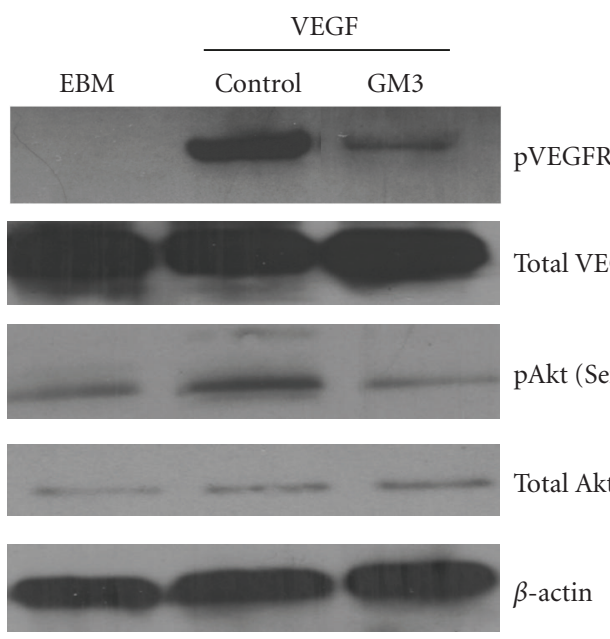

(a)

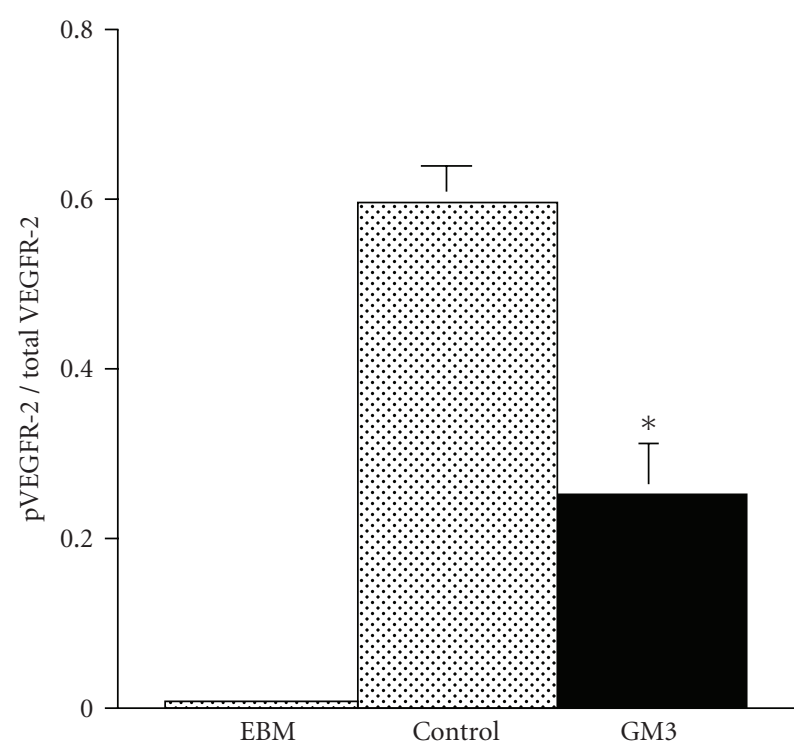

(b)

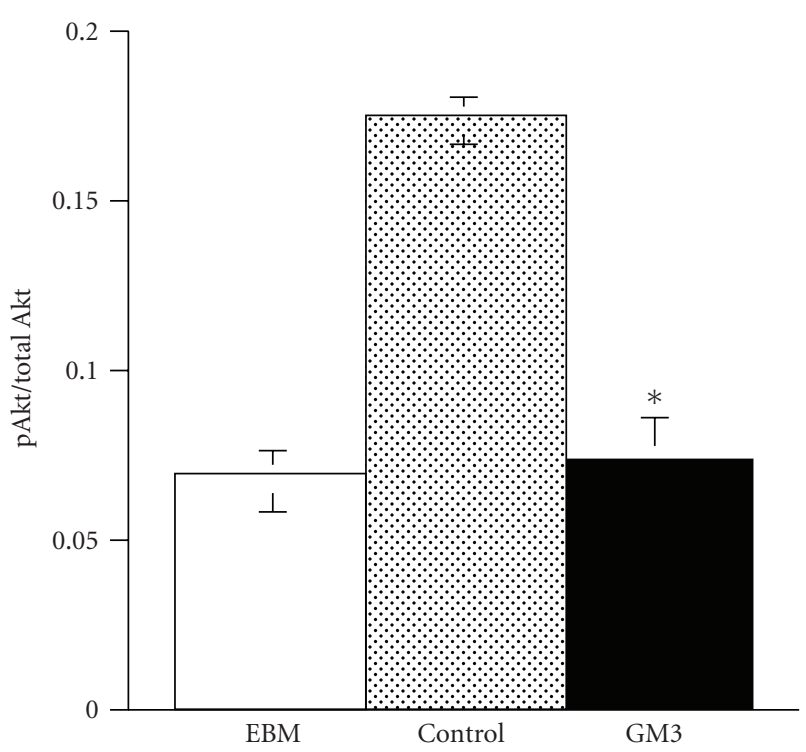

(c)

FIGURE 8: GM3 inhibits VEGFR-2 and Akt phosphorylation in HUVEC. HUVECs were incubated with GM3 (100 ng/ml) in endothelial basal medium (EBM) for 24 hours and were then stimulated with VEGF (100 ng/ml) for 5 minutes as we described [49]. (a) Cell lysates were prepared and measurement of VEGFR-2 and Akt phosphorylation over total was analyzed using Western blots [49]. (b) VEGFR-2 and (c) Akt phosphorylation were significantly lower in GM3-treated HUVEC than in control HUVEC $(P<.001)$. Values are expressed as means \pm SEM ( $n=3$ independent experiments).

In summary, our results show that GM3 inhibits brain tumor angiogenesis. GM3 targets both tumor cells and endothelial cells. Although GM3 is elevated in human malignant brain tumors, its concentration is less than that of complex gangliosides especially GD3. We suggest that increasing the ratio of GM3 to complex gangliosides may be effective in reducing angiogenesis and growth in human glioblastomas. Our findings suggest that pharmacological application of GM3 is warranted as a potential nontoxic anti-angiogenic therapy for malignant brain cancer.

\section{References}

[1] J. K. Lowry, J. J. Snyder, and P. W. Lowry, "Brain tumors in the elderly: recent trends in a Minnesota cohort study," Archives of Neurology, vol. 55, no. 7, pp. 922-928, 1998.

[2] J. Kaiser, "No meeting of minds on childhood cancer," Science, vol. 286, no. 5446, pp. 1832-1834, 1999. 
[3] P. Kaatsch, C. H. Rickert, J. Kühl, J. Schüz, and J. Michaelis, "Population-based epidemiologic data on brain tumors in German children,” Cancer, vol. 92, no. 12, pp. 3155-3164, 2001.

[4] P. J. Jukich, B. J. McCarthy, T. S. Surawicz, S. Freels, and F. G. Davis, "Trends in incidence of primary brain tumors in the United States, 1985-1994," Neuro-Oncology, vol. 3, no. 3, pp. 141-151, 2001.

[5] H. Sontheimer, "A role for glutamate in growth and invasion of primary brain tumors," Journal of Neurochemistry, vol. 105, no. 2, pp. 287-295, 2008.

[6] C.-L. Tso, P. Shintaku, J. Chen, et al., "Primary glioblastomas express mesenchymal stem-like properties," Molecular Cancer Research, vol. 4, no. 9, pp. 607-619, 2006.

[7] L. E. Abate, P. Mukherjee, and T. N. Seyfried, "Gene-linked shift in ganglioside distribution influences growth and vascularity in a mouse astrocytoma," Journal of Neurochemistry, vol. 98, no. 6, pp. 1973-1984, 2006.

[8] D. Zagzag, M. Esencay, O. Mendez, et al., "Hypoxia- and vascular endothelial growth factor-induced stromal cell-derived factor- $1 \alpha /$ CXCR4 expression in glioblastomas: one plausible explanation of Scherer's structures," American Journal of Pathology, vol. 173, no. 2, pp. 545-560, 2008.

[9] M. Kirsch, G. Schackert, and P. M. Black, "Anti-angiogenic treatment strategies for malignant brain tumors," Journal of Neuro-Oncology, vol. 50, no. 1-2, pp. 149-163, 2000.

[10] H. Bai, J. Orlando, and T. N. Seyfried, "Altered ganglioside composition in virally transformed rat embryo fibroblast," Biochimica et Biophysica Acta, vol. 1136, no. 1, pp. 23-27, 1992.

[11] M. El-Abbadi, T. N. Seyfried, A. J. Yates, C. Orosz, and M. C. Lee, "Ganglioside composition and histology of a spontaneous metastatic brain tumour in the VM mouse," British Journal of Cancer, vol. 85, no. 2, pp. 285-292, 2001.

[12] S. Hakomori, "Glycosylation defining cancer malignancy: new wine in an old bottle," Proceedings of the National Academy of Sciences of the United States of America, vol. 99, no. 16, pp. 10231-10233, 2002.

[13] H.-J. Choi, T.-W. Chung, S.-K. Kang, et al., "Ganglioside GM3 modulates tumor suppressor PTEN-mediated cell cycle progression-transcriptional induction of $\mathrm{p} 21^{\mathrm{WAF} 1}$ and p $27^{\text {kip1 }}$ by inhibition of PI-3K/AKT pathway," Glycobiology, vol. 16, no. 7, pp. 573-583, 2006.

[14] H. Bai and T. N. Seyfried, "Influence of ganglioside GM3 and high density lipoprotein on the cohesion of mouse brain tumor cells," Journal of Lipid Research, vol. 38, no. 1, pp. 160172, 1997.

[15] A. J. Yates and A. Rampersaud, "Sphingolipids as receptor modulators: an overview," Annals of the New York Academy of Sciences, vol. 845, pp. 57-71, 1998.

[16] T. N. Seyfried, R. K. Yu, M. Saito, and M. Albert, "Ganglioside composition of an experimental mouse brain tumor," Cancer Research, vol. 47, no. 13, pp. 3538-3542, 1987.

[17] J. A. Ecsedy, K. A. Holthaus, H. C. Yohe, and T. N. Seyfried, "Expression of mouse sialic acid on gangliosides of a human glioma grown as a xenograft in SCID mice," Journal of Neurochemistry, vol. 73, no. 1, pp. 254-259, 1999.

[18] H.-H. Chou, H. Takematsu, S. Diaz, et al., "A mutation in human CMP-sialic acid hydroxylase occurred after the Homo-Pan divergence," Proceedings of the National Academy of Sciences of the United States of America, vol. 95, no. 20, pp. 11751-11756, 1998.

[19] E. A. Muchmore, M. Milewski, A. Varki, and S. Diaz, "Biosynthesis of N-glycolyneuraminic acid. The primary site of hydroxylation of $\mathrm{N}$-acetylneuraminic acid is the cytosolic sugar nucleotide pool," Journal of Biological Chemistry, vol. 264, no. 34, pp. 20216-20223, 1989.

[20] I. Kawashima, H. Ozawa, M. Kotani, et al., "Characterization of ganglioside expression in human melanoma cells: immunological and biochemical analysis," Journal of Biochemistry, vol. 114, no. 2, pp. 186-193, 1993.

[21] M. El-Abbadi and T. N. Seyfried, "Influence of growth environment on the ganglioside composition of an experimental mouse brain tumor," Molecular and Chemical Neuropathology, vol. 21, no. 2-3, pp. 273-285, 1994.

[22] M. Ziche, G. Alessandri, and P. M. Gullino, "Gangliosides promote the angiogenic response," Laboratory Investigation, vol. 61, no. 6, pp. 629-634, 1989.

[23] W. Song, M. F. Vacca, R. Welti, and D. A. Rintoul, "Effects of gangliosides $\mathrm{G}_{\mathrm{M} 3}$ and de- $\mathrm{N}$-acetyl $\mathrm{G}_{\mathrm{M} 3}$ on epidermal growth factor receptor kinase activity and cell growth," Journal of Biological Chemistry, vol. 266, no. 16, pp. 10174-10181, 1991.

[24] A. Rebbaa, J. Hurh, H. Yamamoto, D. S. Kersey, and E. G. Bremer, "Ganglioside GM3 inhibition of EGF receptor mediated signal transduction," Glycobiology, vol. 6, no. 4, pp. 399-406, 1996.

[25] K. Kabayama, T. Sato, F. Kitamura, et al., "TNA $\alpha$-induced insulin resistance in adipocytes as a membrane microdomain disorder: involvement of ganglioside GM3," Glycobiology, vol. 15, no. 1, pp. 21-29, 2005.

[26] S. Tagami, J.-I. Inokuchi, K. Kabayama, et al., "Ganglioside GM3 participates in the pathological conditions of insulin resistance," Journal of Biological Chemistry, vol. 277, no. 5, pp. 3085-3092, 2002.

[27] E. G. Bremer, J. Schlessinger, and S. Hakomori, "Gangliosidemediated modulation of cell growth. Specific effects of $\mathrm{GM}_{3}$ on tyrosine phosphorylation of the epidermal growth factor receptor," Journal of Biological Chemistry, vol. 261, no. 5, pp. 2434-2440, 1986.

[28] M. S. Toledo, E. Suzuki, K. Handa, and S. Hakomori, "Effect of ganglioside and tetraspanins in microdomains on interaction of integrins with fibroblast growth factor receptor," Journal of Biological Chemistry, vol. 280, no. 16, pp. 16227-16234, 2005.

[29] Y. Nakatsuji and R. H. Miller, "Selective cell-cycle arrest and induction of apoptosis in proliferating neural cells by ganglioside GM3," Experimental Neurology, vol. 168, no. 2, pp. 290-299, 2001.

[30] G. Alessandri, K. S. Raju, and P. M. Gullino, "Interaction of gangliosides with fibronectin in the mobilization of capillary endothelium. Possible influence on the growth of metastasis," Invasion and Metastasis, vol. 6, no. 3, pp. 145-165, 1986.

[31] G. Alessandri, P. Cornaglia-Ferraris, and P. M. Gullino, "Angiogenic and angiostatic microenvironment in tumors: role of gangliosides," Acta Oncologica, vol. 36, no. 4, pp. 383387, 1997.

[32] M. Ziche, L. Morbidelli, G. Alessandri, and P. M. Gullino, "Angiogenesis can be stimulated or repressed in vivo by a change in GM3:GD3 ganglioside ratio," Laboratory Investigation, vol. 67, no. 6, pp. 711-715, 1992.

[33] G. Alessandri, S. Filippeschi, P. Sinibaldi, et al., "Influence of gangliosides on primary and metastatic neoplastic growth in human and murine cells," Cancer Research, vol. 47, no. 16, pp. 4243-4247, 1987.

[34] M. G. Manfredi, S. Lim, K. P. Claffey, and T. N. Seyfried, “Gangliosides influence angiogenesis in an experimental mouse brain tumor," Cancer Research, vol. 59, no. 20, pp. 5392-5397, 1999. 
[35] M. H. Ravindranath, T. Tsuchida, D. L. Morton, and R. F. Irie, "Ganglioside GM3:GD3 ratio as an index for the management of melanoma," Cancer, vol. 67, no. 12, pp. 3029-3035, 1991.

[36] R. Jennemann, H.-D. Mennel, B. L. Bauer, and H. Wiegandt, "Glycosphingolipid component profiles of human gliomas correlate with histological tumour types: analysis of inter-individual and tumour-regional distribution," Acta Neurochirurgica, vol. 126, no. 2-4, pp. 170-178, 1994.

[37] S. Koochekpour and G. J. Pilkington, "Vascular and perivascular GD3 expression in human glioma," Cancer Letters, vol. 104, no. 1, pp. 97-102, 1996.

[38] P. Fredman, H. von Holst, V. P. Collins, B. Dellheden, and L. Svennerholm, "Expression of gangliosides GD3 and 3'isoLM1 in autopsy brains from patients with malignant tumors," Journal of Neurochemistry, vol. 60, no. 1, pp. 99-105, 1993.

[39] B. Berra, S. M. Gaini, and L. Riboni, "Correlation between ganglioside distribution and histological grading of human astrocytomas," International Journal of Cancer, vol. 36, no. 3, pp. 363-366, 1985.

[40] T. D. Traylor and E. L. Hogan, "Gangliosides of human cerebral astrocytomas," Journal of Neurochemistry, vol. 34, no. 1, pp. 126-131, 1980.

[41] G. Zeng, L. Gao, S. Birkle, and R. K. Yu, "Suppression of ganglioside GD3 expression in a rat F-11 tumor cell line reduces tumor growth, angiogenesis, and vascular endothelial growth factor production," Cancer Research, vol. 60, no. 23, pp. 6670-6676, 2000.

[42] L. Manuelidis, R. K. Yu, and E. E. Manuelidis, "Ganglioside content and pattern in human gliomas in culture. Correlation of morphological changes with altered gangliosides," Acta Neuropathologica, vol. 38, no. 2, pp. 129-135, 1977.

[43] L. C. Huysentruyt, P. Mukherjee, D. Banerjee, L. M. Shelton, and T. N. Seyfried, "Metastatic cancer cells with macrophage properties: evidence from a new murine tumor model," International Journal of Cancer, vol. 123, no. 1, pp. 73-84, 2008.

[44] T. N. Seyfried, M. El-Abbadi, and M. L. Roy, "Ganglioside distribution in murine neural tumors," Molecular and Chemical Neuropathology, vol. 17, no. 2, pp. 147-167, 1992.

[45] P. Mukherjee, L. E. Abate, and T. N. Seyfried, "Antiangiogenic and proapoptotic effects of dietary restriction on experimental mouse and human brain tumors," Clinical Cancer Research, vol. 10 , no. 16, pp. 5622-5629, 2004.

[46] E. G. Bremer and S. I. Hakomori, "GM3 ganglioside induces hamster fibroblast growth inhibition in chemically-defined medium: ganglioside may regulate growth factor receptor function," Biochemical and Biophysical Research Communications, vol. 106, no. 3, pp. 711-718, 1982.

[47] T.-W. Chung, S.-J. Kim, H.-J. Choi, et al., "Ganglioside GM3 inhibits VEGF/VEGFR-2-mediated angiogenesis: direct interaction of GM3 with VEGFR-2," Glycobiology, vol. 19, no. 3, pp. 229-239, 2009.

[48] E. N. E. Noll, J. Lin, Y. Nakatsuji, R. H. Miller, and P. M. Black, "GM3 as a novel growth regulator for human gliomas," Experimental Neurology, vol. 168, no. 2, pp. 300-309, 2001.

[49] P. Mukherjee, A. C. Faber, L. M. Shelton, R. C. Baek, T. C. Chiles, and T. N. Seyfried, "Ganglioside GM3 suppresses the proangiogenic effects of vascular endothelial growth factor and ganglioside GD1A," Journal of Lipid Research, vol. 49, no. 5, pp. 929-938, 2008.

[50] H. M. Zimmerman and H. Arnold, "Experimental brain tumors: I. tumors produced with methylcholanthrene," Cancer Research, vol. 1, pp. 919-938, 1941.
[51] J. Marsh, P. Mukherjee, and T. N. Seyfried, "Akt-dependent proapoptotic effects of dietary restriction on late-stage management of a phosphatase and tensin homologue/tuberous sclerosis complex 2-deficient mouse astrocytoma," Clinical Cancer Research, vol. 14, no. 23, pp. 7751-7762, 2008.

[52] R. Martínez-Murillo and A. Martínez, "Standardization of an orthotopic mouse brain tumor model following transplantation of CT-2A astrocytoma cells," Histology and Histopathology, vol. 22, no. 12, pp. 1309-1326, 2007.

[53] J. Jośko and M. Mazurek, "Transcription factors having impact on vascular endothelial growth factor (VEGF) gene expression in angiogenesis," Medical Science Monitor, vol. 10, no. 4, pp. RA89-RA98, 2004.

[54] J. Fang, M. Ding, L. Yang, L.-Z. Liu, and B.-H. Jiang, "PI3K/PTEN/AKT signaling regulates prostate tumor angiogenesis," Cellular Signalling, vol. 19, no. 12, pp. 2487-2497, 2007.

[55] H. D. Skinner, J. Z. Zheng, J. Fang, F. Agani, and B.-H. Jiang, "Vascular endothelial growth factor transcriptional activation is mediated by hypoxia-inducible factor $1 \alpha$, HDM2, and p70S6K1 in response to phosphatidylinositol 3-kinase/AKT signaling," Journal of Biological Chemistry, vol. 279, no. 44, pp. 45643-45651, 2004.

[56] O. Nakamura, M. Iwamori, M. Matsutani, and K. Takakura, "Ganglioside GD3 shedding by human gliomas," Acta Neurochirurgica, vol. 109, no. 1-2, pp. 34-36, 1991.

[57] K. Kaucic, Y. Liu, and S. Ladisch, "Modulation of growth factor signaling by gangliosides: positive or negative?" Methods in Enzymology, vol. 417, pp. 168-185, 2006.

[58] S. Ladisch, S. Kitada, and E. F. Hays, "Gangliosides shed by tumor cells enhance tumor formation in mice," Journal of Clinical Investigation, vol. 79, no. 6, pp. 1879-1882, 1987.

[59] R. Olshefski and S. Ladisch, "Intercellular transfer of shed tumor cell gangliosides," FEBS Letters, vol. 386, no. 1, pp. 1114, 1996.

[60] Y. Liu, R. Li, and S. Ladisch, "Exogenous ganglioside GDla enhances epidermal growth factor receptor binding and dimerization," Journal of Biological Chemistry, vol. 279, no. 35, pp. 36481-36489, 2004.

[61] Z. Lang, M. Guerrera, R. Li, and S. Ladisch, "Ganglioside GD1a enhances VEGF-induced endothelial cell proliferation and migration," Biochemical and Biophysical Research Communications, vol. 282, no. 4, pp. 1031-1037, 2001.

[62] H. van Cruijsen, M. Ruiz, P. van der Valk, T. D. de Gruijl, and G. Giaccone, "Tissue micro array analysis of ganglioside Nglycolyl GM3 expression and signal transducer and activator of transcription (STAT)-3 activation in relation to dendritic cell infiltration and microvessel density in non-small cell lung cancer," BMC Cancer, vol. 9, article 180, 2009. 


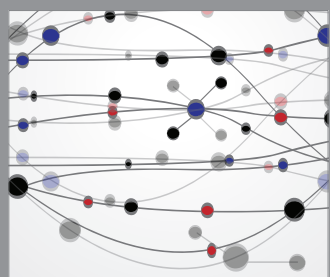

The Scientific World Journal
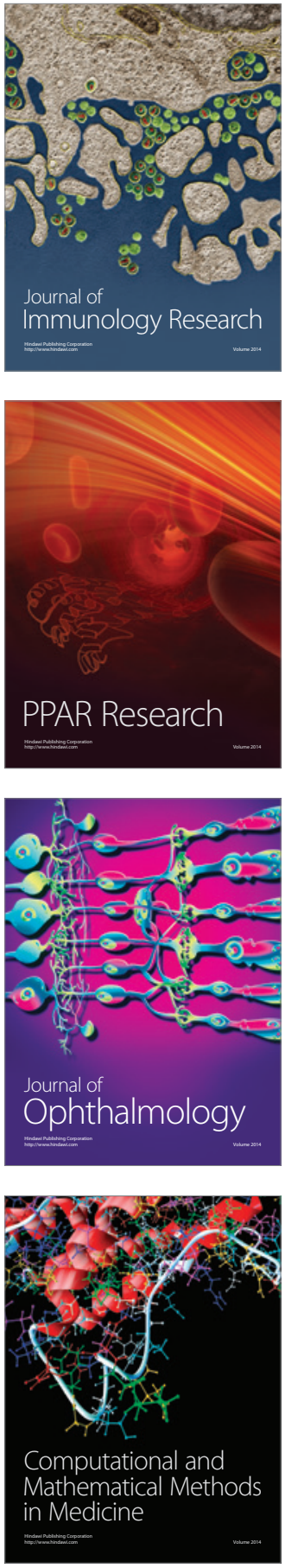

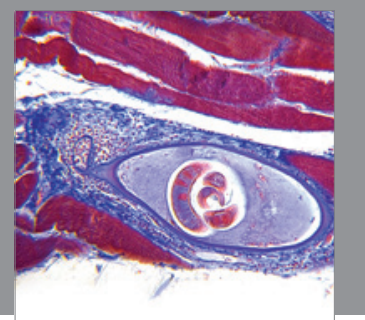

Gastroenterology

Research and Practice
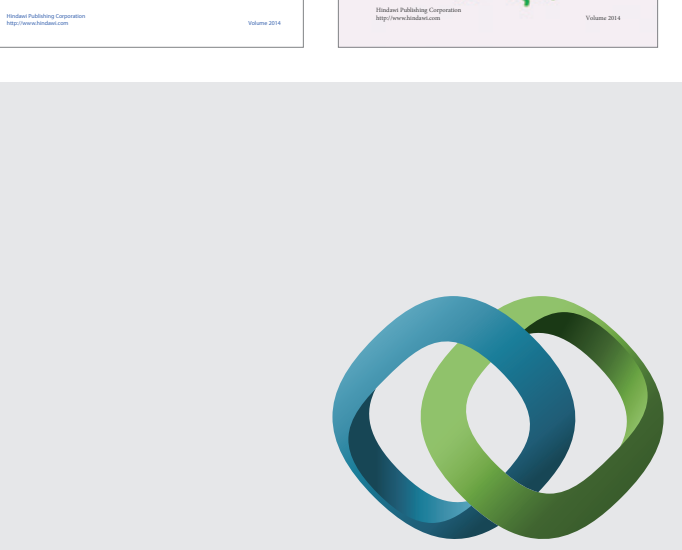

\section{Hindawi}

Submit your manuscripts at

http://www.hindawi.com
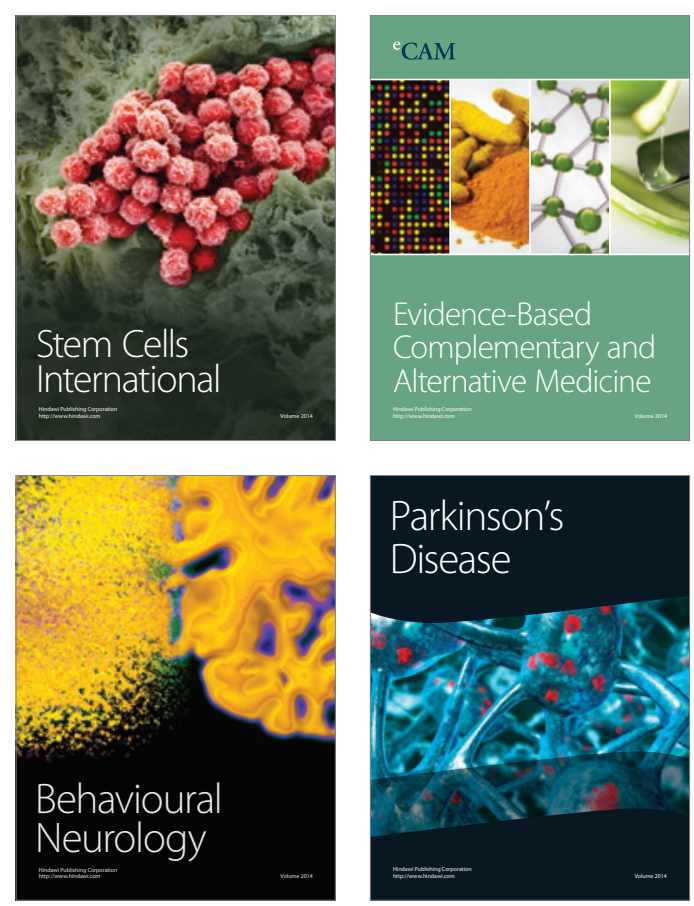

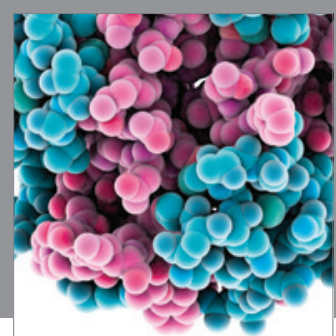

Journal of
Diabetes Research

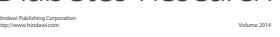

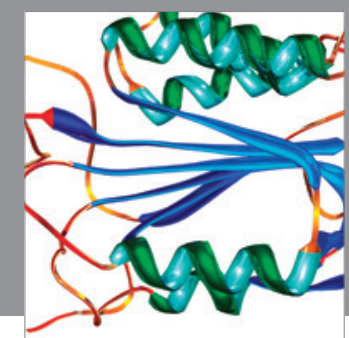

Disease Markers
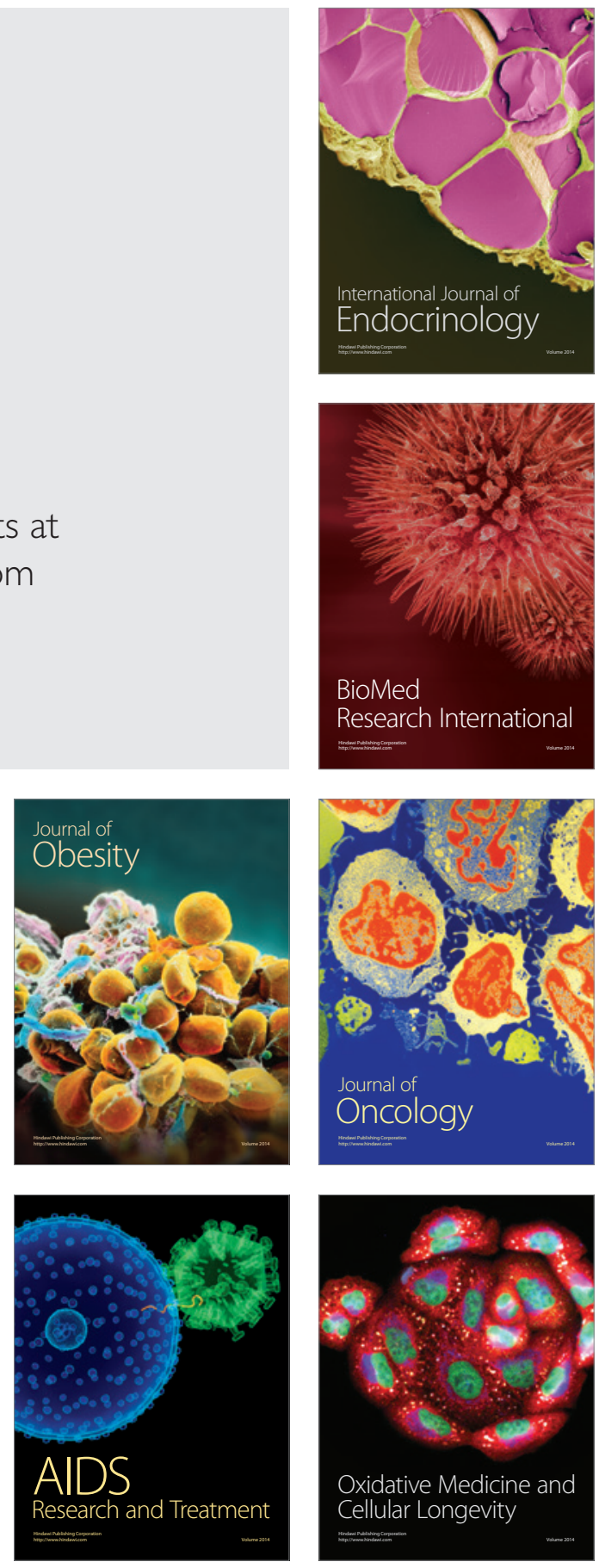